\title{
Limits to load-lifting performance in a passerine bird: the effects of intraspecific variation in morphological and kinematic parameters
}

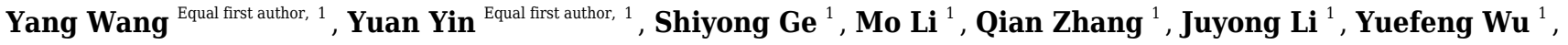 \\ Dongming Li ${ }^{\text {Corresp., } 1}$, Robert Dudley ${ }^{2}$ \\ ${ }^{1}$ Key Laboratory of Animal Physiology, Biochemistry and Molecular Biology of Hebei Province, College of Life Sciences, Hebei Normal University, \\ Shijiazhuang, Hebei, China \\ 2 Department of Integrative Biology, University of California, Berkeley, Berkeley, California, United States \\ Corresponding Author: Dongming Li \\ Email address: lidongming@hebtu.edu.cn
}

Although more massive flight muscles along with larger wings, higher wingbeat frequencies, and greater stroke amplitudes enhance force and power production in flapping flight, the extent to which these parameters may be correlated with other morphological features relevant to flight physiology and biomechanics remains unclear. Intraspecifically, we hypothesized that greater vertical load-lifting capacity would correlate with higher wingbeat frequencies and relatively more massive flight muscles, along with relatively bigger hearts, lungs, and stomachs to enhance metabolic capacity and energy supply, but also with smaller body size given the overall negative allometric dependence of maximum flight performance in volant taxa. To explore intraspecific correlates of flight performance, we assembled a large dataset that included thirteen morphological and kinematic variables for a non-migratory passerine, the Eurasian tree sparrow (Passer montanus). We found that heavier flight muscles and larger wings, heavier stomachs, and shorter bills were the most important correlates of maximum load-lifting capacity. Surprisingly, wingbeat frequency, wing stroke amplitude, and masses of the heart, lungs, and digestive organs (except for the stomach) were non-significant predictor variables relative to lifting capacity. The best-fit structural equation model indicated that load-lifting capacity was positively correlated with flight muscle mass, wing area, and stomach mass, but was negatively correlated with bill length. Characterization of individual variability in flight performance in a free-ranging passerine indicates the subtlety of interaction effects among morphological features, some of which differ from those that have been identified interspecifically for maximum flight performance in birds. 
1 Manuscript Title

2 Limits to load-lifting performance in a passerine bird: the effects of

3 intraspecific variation in morphological and kinematic parameters

4

5 Yang Wang ${ }^{1, \dagger}$, Yuan Yin ${ }^{1, \dagger}$, Shiyong $\mathrm{Ge}^{1}, \mathrm{Mo} \mathrm{Li}^{1}$, Qian Zhang ${ }^{1}$, Juyong $\mathrm{Li}^{1}$, Yuefeng $\mathrm{Wu}^{1}$, Dongming

$6 \quad \mathrm{Li}^{1, *}$ and Robert Dudley ${ }^{2}$

$7 \quad{ }^{1}$ Key Laboratory of Animal Physiology, Biochemistry and Molecular Biology of Hebei

8 Province, College of Life Sciences, Hebei Normal University, Shijiazhuang 050024, P. R. China

$9 \quad{ }^{2}$ Department of Integrative Biology, University of California, Berkeley, CA 94720, USA

$11 \dagger$ These authors contributed equally to this work.

Running title: Maximum load-lifting capacity in sparrows

*Corresponding author:

Dongming Li1 E-mail: lidngmng@gmail.com, telephone: 86-311-80787556, fax: 86-311-

$17 \quad 80787555$ 
Abstract

Although more massive flight muscles along with larger wings, higher wingbeat frequencies, and greater stroke amplitudes enhance force and power production in flapping flight, the extent to which these parameters may be correlated with other morphological features relevant to flight physiology and biomechanics remains unclear. Intraspecifically, we hypothesized that greater vertical load-lifting capacity would correlate with higher wingbeat frequencies and relatively more massive flight muscles, along with relatively bigger hearts, lungs, and stomachs to enhance metabolic capacity and energy supply, but also with smaller body size given the overall negative allometric dependence of maximum flight performance in volant taxa. To explore intraspecific correlates of flight performance, we assembled a large dataset that included thirteen morphological and kinematic variables for a non-migratory passerine, the Eurasian tree sparrow (Passer montanus). We found that heavier flight muscles and larger wings, heavier stomachs, and shorter bills were the most important correlates of maximum load-lifting capacity. Surprisingly, wingbeat frequency, wing stroke amplitude, and masses of the heart, lungs, and digestive organs (except for the stomach) were non-significant predictor variables relative to lifting capacity. The best-fit structural equation model indicated that load-lifting capacity was positively correlated with flight muscle mass, wing area, and stomach mass, but was negatively correlated with bill length. Characterization of individual variability in flight performance in a free-ranging passerine indicates the subtlety of interaction effects among morphological features, some of which differ from those that have been identified interspecifically for maximum flight performance in birds.

Keywords: Eurasian tree sparrow, flight performance, kinematics, maximum load-lifting capacity 


\section{Introduction}

Birds exhibit a broad diversity of flight-related morphological and physiological characteristics (Hedenström, 2002; Lee et al., 2014; Puttick et al., 2014; Altshuler et al., 2015; Butler, 2016), many of which reflect multiple trade-offs in flight performance (see Goslow et al., 1990; Ellington, 1991; Chai and Dudley, 1999; Lind 2001; Lind \& Jakobsson 2001; Altshuler et al., 2015). For example, larger species typically possess bigger wings and higher pectoral muscle mass, whereas wingbeat frequency declines with increasing body mass (e.g., Groom et al., 2018); higher wingbeat frequencies and greater stroke amplitudes nonetheless yield increased force and power production (e.g., Chai and Dudley, 1995; Hedenström, 2002; Altshuler et al., 2015). Wing morphological and kinematic features along with flight-related muscle, are thus key variables influencing flight performance.

Avian flight is an energy-demanding activity requiring powerful respiratory and cardiovascular systems to support the intense metabolism of the associated skeletal muscles (Hedenström, 2002; Lee et al., 2014; Altshuler et al., 2015; Butler, 2016; Nespolo et al., 2018). As major metabolic engines, heart and lung capacities underpin both burst power and endurance flight performance (see Bishop and Butler, 1995; Wright et al., 2014). Moreover, larger nutritional organs and increases in the quantity of digestive enzymes and nutrient transporters are essential to meet the high-energy demands of flight (Karasov and McWilliams, 2005). For example, many birds exhibited a reduction in the pectoralis primary mass as a consequence of lowered nutritional supplies in post-migratory periods (Dietz et al., 2007; Piersma and Dietz, 2007). Therefore, respiratory, cardiovascular, and nutritional systems represent important features for sustaining powered flight.

Other morphological factors contributing to an increase in body mass could be considered as hindering features that reduce available power for flight (Ellington, 1991; Dial, 2003). For example, a toothless beak in extant birds is believed to increase flight efficiency by reducing overall body mass (Louchart and Viriot, 2011). However, relationships among diverse 
68

69

morphological features relative to flight capability in free-living birds have not been well investigated.

Maximum load-lifting capacity (as imposed via asymptotic loading; Buchwald and Dudley, 2010), is an informative means of evaluating burst capacity in volant taxa (e.g., Altshuler et al., 2004; 2010; Sun et al., 2016). To date, interspecific comparisons have evaluated morphological and functional correlates of the maximum load-lifting capacity of free-living birds (see Hedenström, 2002; Lee et al., 2014; Altshuler et al., 2015; Butler, 2016; Groom et al., 2018; Nespolo et al., 2018), however, much less information is available about intraspecific determinants of maximum loading-lifting capacity. Intraspecifically, we hypothesize that maximum load-lifting capacity will be positively influenced by key morphological and kinematic features relevant to force and power production (e.g., relatives size of flight muscles and the wings, wingbeat frequency, and stroke amplitude), as well as by metabolically relevant features (including hearts, lungs, and digestive organs relevant to sustained flight).

To evaluate these hypotheses, we assembled a large dataset with a total of 13 variables of morphological, internal anatomical, and biomechanical variables for a non-migrant passerine, namely the Eurasian tree sparrow (Passer montanus). We first determined statistically the most important variables contributing to maximum load-lifting capacity (total lifted load, i.e., the sum of body mass and the maximum supplemental load), as determined by asymptotic load-lifting experiments. We further assessed the relative contribution of each variable to maximum loadlifting capacity, then identified the most critical factors influencing intraspecific variation using multiple-variable interactions and structural equation model (SEM) in this transient feature of flight performance. 
90

91

92

93

94

95

96

97

98

99

100

101

102

103

104

105

106

107

108

109

110

111

112

113

114

115

\section{Materials \& Methods}

\section{Bird collection}

Totals of 33 male and 39 female adult Eurasian tree sparrows were captured opportunistically using mist nets during the late winter of 2017 (i.e., 13 March to 1 April) at five different lowland sites (Dongyangshi: N37.9667, E114.602 ${ }^{\circ}$; Yuhuaqu: N38.021 ${ }^{\circ}, \mathrm{E} 114.526^{\circ}$;

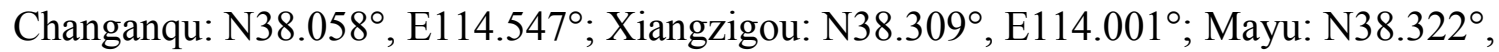
E113.962 ${ }^{\circ}$; site elevational range from 80 - 203 m) around Shijiazhuang City, Hebei Province, People's Republic of China. Within 30 min post-capture, body mass to within $0.01 \mathrm{~g}$ was measured for each bird using a portable digital balance.

\section{Load-lifting assay}

Birds were placed individually (within 3 hours of capture) in a rectangular flight chamber $(45 \mathrm{~cm} \times 45 \mathrm{~cm} \times 150 \mathrm{~cm})$ made from transparent acrylic sheet, as used in previous experiments (Sun et al., 2016). Each bird was evaluated for asymptotic load-lifting capacity using an assay described in detail elsewhere (see Sun et al., 2016). Briefly, a thread with different plastic beads (each approximately $1.0 \mathrm{~g}$ in mass) and positioned at fixed linear intervals was attached to the left tarsometatarsus of the sparrow. When released from the bottom of the chamber, birds typically flew vertically towards the top, asymptotically lifting more and more beads until a maximum load was attained. Two cameras were used in this experiment; one high-speed video camera (JVC GCP100BAC; operated at 50 frames $^{-1}$; see Supplemental material) positioned laterally at a distance of $80 \mathrm{~cm}$ to the chamber was used to film the beads remaining on the chamber floor during maximum load-lifting flight, and thus by subtraction to determine the total extra weight lifted by the bird. The other synchronized camera (JVC GCP100BAC, operated at 250 frames $^{-1}$; see Supplemental material), was positioned laterally near the top of the chamber (see Sun et al., 2016) and was used to obtain wingbeat frequency and stroke amplitude. Multiple ascending flights were recorded for each bird (mean of 4.7 flights), and the maximum weight lifted within the series was assumed to indicate the limit to load-lifting flight 
116

117

118

119

120

121

122

123

124

125

126

127

128

129

130

131

132

133

134

135

136

137

138

139

140

141

performance. Wingbeat frequency was determined from the number of frames required to complete an integral number of wingbeats for a composite sequence containing multiple flapping cycles, but starting and finishing at the same vertical position of the wings. Wing stroke amplitude was calculated as the angle between extreme wing tip positions (i.e., the point of the outermost primary feather relative to the longitudinal body axis) at extremes of the nominally vertical wing stroke, as filmed by the top lateral camera (see Sun et al., 2016). A mean value for stroke amplitude was calculated from three to five separate measurements within each bout of maximum load within the final $0.5 \mathrm{~s}$ of peak lifting.

\section{Morphological, anatomical, and kinematic parameters}

To identify potential influences total lifted load, we determined for each individual bird a total of 13 variables of morphological (sex, body mass, bill length, total wing area, aspect ratio), internal anatomical (the masses of flight muscle, heart, lung, liver, stomach, and gut length), and biomechanical relevance (wingbeat frequency and amplitude). Following load-lifting experiments, each bird was immediately euthanized with phenobarbitone $\left(7.5 \mu \mathrm{g} \mathrm{g}^{-1}\right.$ body mass), and its bill length was measured to the nearest $0.1 \mathrm{~mm}$ using Vernier calipers. The right wing was photographed for measurements of wing area and wing length $R$ (analyzed using ImageJ, National Institutes of Health, Bethesda, MD, USA); total wing area $S$ is given by twice the area of the right wing. The aspect ratio is given by $(2 R)^{2} / S$. The pectoralis major, pectoralis minor, and the whole heart, lung, liver (all following blotting to remove blood), along with the fresh gut and stomach (food residue was removed by washing with water), were then excised and weighed using a digital balance sensitive to $0.1 \mathrm{mg}$ (with gut length measured to $\pm 1 \mathrm{~mm}$ ). All protocols were approved by the Ethics and Animal Welfare Committee (no. 2013-6) and by the Institutional Animal Care and Use Committee (HEBTU2013-7) of Hebei Normal University, China, and were carried out under the auspices of scientific collecting permits issued by the Department of Wildlife Conservation (Forestry Bureau) of Hebei Province, China.

\section{Statistical analyses}


We calculated means and standard deviations for gross morphological (body mass, bill length, total wing area, aspect ratio), internal anatomical (the masses of flight muscle, heart, lung, liver, stomach, and gut length), and kinematic variables (wingbeat frequency and wing stroke amplitude). We determined Pearson correlations between maximum load-lifting flight performance (i.e., total lifted load) and all other variables, including sex as a discrete covariate. We then implemented a generalized linear model (GLM) using the glm function in Program R v. 3.4.2 (Pinheiro et al., 2015) to model relationships between dependent factors (i.e., total lifted load) and all independent variables, with sex as a discrete covariate. All continuous variables were scaled (i.e., centralized and standardized; Schielzeth, 2010) before such modeling to reduce multicollinearity. Multiparameter models were discarded if a nested model (i.e., collinearity among factors) containing a subset of the same parameters had a better Akaike's Information Criterion (AIC) score. To account for model selection uncertainty, model-averaged estimates of variable coefficients were computed using the 'best model set', defined as the set of models for which delta AIC was less than 6 (see Burnham and Anderson, 2002). All possible models were averaged to identify the most important variables, using the importance score in the MuMIn package (Kamil, 2013) of Program R v.3.4.2. We ranked all variables selected by the average model, and then considered those variables with a higher relative importance score (i.e., $>0.7$ ) as determinant variables for total lifted load. We further assessed whether there were level-two interaction effects on maximum load-lifting flight performance, and then selected those most important variables underlying variance in total lifted load by identifying those with relative importance scores $>0.7$. Finally, we constructed a SEM in the lavaan package (Rosseel, 2012) of Program R v.3.4.2, including all combinations of those important variables as identified by AIC scores, and selected the best model with a Chi-square test, the root mean square error of approximation (RMSEA), the standard root mean square residual (SRMR), and the comparative fit index (CFI). We assumed a well-fitted model to have a p-value $>0.05$ for the Chi-square test, a RMSEA and SRMR test with values less than 0.1, and a CFI close to 1 (i.e., >0.9). The 
relationships between measured variables and total lifted load were represented by regression coefficients; all path coefficients used standardized estimates.

\section{Results}

Total lifted load was positively correlated with diverse traits, including body mass, wing area, the masses of flight muscle, heart, lung, liver, stomach, and length of gut (Table 1). Among all measured variables, variation in total lifted load was best explained by variability in bill length, stomach mass, gut length, wing area, and flight muscle mass (Table 2). However, sex and other morphological (body mass, aspect ratio), internal anatomical (masses of heart, lung, and liver), and kinematic features (wingbeat frequency and wing stroke amplitude) did not significantly predict variation in maximum load-lifting capacity (Table 2). Specifically, heavier flight muscles, a greater stomach mass, larger wings, and a longer gut but also shorter bills were strongly correlated with total lifted load. Among these five variables, flight muscle mass, bill length, wing area, and stomach mass were the four most important factors predicting total lifted load when all level-two interactions were considered (Table 3).

The best SEM consisting of the four most important variables ,including flight muscle mass, bill length, wing area, and stomach mass, was well-fitted $\left(\chi^{2}=3.21, d f=2, p=0.201\right.$; RMSEA $=$ $0.092, \mathrm{SRMR}=0.071, \mathrm{CFI}=0.983$; see Figure 1$)$. Total lifted load was positively correlated with sizes of the flight muscles and wings, and with stomach mass, but was negatively correlated with bill length (Table S1, Figure 1). We also found masses of the flight muscle to be positively correlated with both bill length and stomach mass (Table S2, Figure 1).

\section{Discussion}

Many external morphological and internal anatomical measurements are positively correlated with maximum load-lifting performance in Eurasian tree sparrows (Table 1), but the strongest correlates are a greater flight muscle mass, larger wings, heavier stomach, and a shorter bill (Table 3), all of which are independent of sex. Interspecifically for birds, wingbeat frequency 
193

194

195

196

197

198

199

200

201

202

203

204

205

206

207

208

209

210

211

212

213

214

215

216

217

218

219

and wing stroke amplitude are key flight kinematic variables for production of aerodynamic power output, along with larger wings (Chai and Dudley, 1999; Hedenström, 2002; Altshuler et al., 2015). By contrast, we here determined that wingbeat frequency and wing stroke amplitude were not strong intraspecific determinants of maximum load-lifting capacity. Variation among individuals in both wing stroke amplitude and wingbeat frequency during maximum load-lifting was small (i.e., less than $3 \%$ and 10\%, respectively; see Table 1). These results are similar to previous findings in Eurasian tree sparrows showing that wing stroke amplitude during maximum load-lifting did not vary across elevational gradients (Sun et al., 2016). Wingbeat frequency did not affect the maximum load-lifting capacity for the lowland populations studied here but did increase in intraspecific comparisons of populations across an altitudinal gradient (Sun et al., 2016). Wingbeat frequency is thus a potential determinant of maximum load-lifting capacity that can vary interspecifically among birds, and that may covary with other morphological traits such as body mass and wing area, but that is relatively invariant within individual populations of these free-living passerines during maximum load-lifting flight. Other flight kinematic parameters, such as downstroke:upstroke ratio, wing angle of attack, and stroke plane angle, may also vary among individuals in maximum load-lifting and warrant further investigation.

In volant taxa, flight muscle mass is an important feature contributing to maximum force production (see Plateau 1865, Marden 1987). Similarly, maximum load-lifting ability (and likely power production as well) in Eurasian tree sparrows is positively influenced by more massive locomotor muscles, but also by wing area (Figure 1, Table 3; see also Lind \& Jakobsson 2001). In general, sustained power production by flight muscle can be constrained by interactions between oxygen supply, substrate availability, and muscle demand from other physiological systems, e.g., the digestive organs or respiratory and circulatory systems (Suarez et al., 1997; Nespolo et al., 2018). However, flight performance during maximum load-lifting by sparrows showed only weak correlations with the sizes of the heart, lung, gut, and liver, but did show a positive correlation with stomach mass (see Table 3; Figure 1). In short-duration flights, oxygen 
220

221

222

223

224

225

226

227

228

229

230

231

232

233

234

235

236

237

238

239

240

241

242

243

244

245

246

and energy supply may not limit such performance, which may be largely anaerobic in character (Altimiras, 2017). Alternatively, aerodynamic force production by the wings can directly constrain whole-animal vertical load-lifting (e.g., Chai et al., 1996), as opposed to limits on power production by the flight muscle. Eurasian tree sparrows are non-migratory and also are a human commensal species (del Hoyo et al., 2017), and as such are much less engaged in substantial lipid loading or digestive tract reduction (as characterizes many long-distance migrants; e.g., Piersma and Gill, 1998). Larger digestive organs may thus indirectly correlate with better burst flight performance in non-migratory avian species. Shorter bills were correlated with higher maximum load-lifting capacity in Eurasian tree sparrows when effects of variable flight muscle mass were incorporated (Table 3; Figure 1), which may reflect unmeasured features of foraging behavior on flight ability, such as differential foraging strategies associated with bill size and matched by changes in flight performance. Bill size also influences heat transfer capacity in some birds (Ryeland et al., 2017; Tattersall et al., 2017), and can limit the suitability of prey items and thus foraging styles (Cruz et al., 2001), so that multiple aspects of bill size may be under selection.

Lift production from the wings of Eurasian tree sparrows may increase with aspect ratio, as in hummingbirds (Kruyt et al., 2014), but a detailed aerodynamic analysis of sparrow takeoff relative to wing design is not available. Although the Eurasian tree sparrow is typically thought of as a sexually monomorphic species, females did have shorter wings and a reduced wing area relative to males (see Mónus et al., 2011; Sun et al., 2016). However, sex was not a determinant of maximum load-lifting capacity, indicating that female sparrows have comparable flight performance relative to males in spite of their smaller wings. How natural selection has enabled females to achieve similar lifting ability is intriguing and warrants further investigation.

\section{Conclusions}

Maximum load-lifting flight performance of individual Eurasian tree sparrows was correlated with multiple morphological factors, including flight muscle mass, wing, and bill 
247 lengths, wing area, and stomach, but was unrelated to sex, various internal anatomical features, 248 and measured wingbeat kinematics. Hypothesized effects of the sizes of flight muscles and wings 249 on maximum load-lifting capacity was confirmed. Kinematic features (wingbeat frequency and 250 wing stroke amplitude) showed no such intraspecific effects, whereas a larger bill and a smaller

251

252

253

254

255

256

257

258

259

260

261

262

263

264

265

stomach compromised flight performance. Overall, the characterization of individual variability in flight performance in a free-living passerine indicates subtlety of interactions among multiple morphological features, some of which differ from those that have been identified interspecifically among birds. Eurasian tree sparrows are also social flockers, and selection on escape performance from the ground may depend in part on group response to a perceived threat. The extent to which vertical accelerations are used in this behavior, and the extent to which other aspects of maneuverability (e.g., rotational ability) are important remain to be investigated. As a human commensal and hyperabundant bird species across the Eurasian continent, such effects are readily amenable to future study under field conditions.

\section{Acknowledgments}

\section{Data Accessibility}

Supplemental information for this article can be found online.

\section{Conflicts of Interest}

The authors declare that there are no conflicts of interest.

\section{Authors' Contributions}

D.L. and R.D. conceived the ideas and designed the study; Y.W., Y.Y., S.G., M.L., Q.Z. and J.L. conducted the experiment and collected the data; Y.W. carried out the statistical analyses with helps of D.L. and Y.W.; D.L., Y.W. and R.D. wrote the manuscript. All authors contributed to the various drafts and gave final approval for publication. 
271 ORCID

272 Yang Wang: https://orcid.org/0000-0001-6626-8278

273 Yuan Yin: https://orcid.org/0000-0002-9661-612X

274 Shiyong Ge: https://orcid.org/0000-0003-0878-3540

275 Mo Li: https://orcid.org/0000-0003-1776-4085

276 Qian Zhang: https://orcid.org/0000-0002-9906-4842

277 Juyong Li: https://orcid.org/0000-0002-7929-427X

278 Yuefeng Wu: https://orcid.org/0000-0001-5121-0049

279 Dongming Li: http://orcid.org/0000-0003-2759-3435

280 Robert Dudley: https://orcid.org/0000-0003-3707-5682 


\section{References}

282 Altimiras, J., Lindgren, I., Giraldo-Deck, Lina María, Matthei, A., \& Garitano-Zavala, álvaro. (2017). Aerobic performance in tinamous is limited by their small heart. A novel hypothesis in the evolution of avian flight. Sci Rep, 7(1), 15964. https://doi.org/10.1038/s41598-017-16297-2

Altshuler, D., Dudley, R., Heredia, S. and McGuire, J. (2010). Allometry of hummingbird lifting performance. J. Exp. Biol. 213, 725-734. https://doi.org/10.1242/jeb.037002

Altshuler, D.L., Dudley, R. and McGuire, J.A. (2004). Resolution of a paradox: hummingbird flight at high elevation does not come without a cost. Proc. Natl. Acad. Sci. USA 101, 17731-17736. https://doi.org/10.1073/pnas.0405260101

Bishop, C. and Butler, P. (1995). Physiological modelling of oxygen consumption in birds during flight. J. Exp. Biol. 98, 2153-2163.

Buchwald, R. and Dudley, R. (2010). Limits to vertical force and power production in bumblebees (Hymenoptera: Bombus impatiens). J. Exp. Biol. 213, 426-432. https://doi.org/10.1242/jeb.033563

Burnham, K. P. and Anderson, D. R. (2002). Model selection and multimodel inference. Springer.

Butler, P.J. (2016) The physiological basis of bird flight. Phil. Trans. Royal Soc. B 371, 1704. https://doi.org/10.1098/rstb.2015.0384

Chai, P. and Dudley, R. (1995). Limits to vertebrate locomotor energetics suggested by hummingbirds hovering in heliox. Nature 377, 722. https://doi.org/10.1086/303179

Chai, P. and Dudley, R. (1999). Maximum flight performance of hummingbirds: capacities, constraints, and trade-offs. Am. Nat. 153, 398-411. https://doi.org/10.1086/303179 
304

305

306

307

308

309

310

311

312

313

314

315

316

317

Chai, P., Harrykissoon, R. and Dudley, R. (1996). Hummingbird hovering performance in hyperoxic heliox: effects of body mass and sex. J. Exp. Biol. 199, 2745-2755. https://doi.org/10.1016/j.phytochem.2007.10.005

Cruz, J.B., Lalas, C., Jillett, J.B., Kitson, J.C., Lyver, P.O.B., Imber, M. and Moller, H. (2001) Prey spectrum of breeding sooty shearwaters (Puffinus griseus) in New Zealand. N. Z. J. Mar. Freshwater Res. 35, 817829. https://doi.org/10.1080/00288330.2001.9517044

del Hoyo, J., Elliott, A., Sargatal, J., Christie, D.A., de Juana, E.E. (2017). Handbook of the Birds of the World Alive. Barcelona: Lynx Edicions (retrieved from www.hbw.com).

Dietz, M.W., Piersma, T., Hedenström, A. and Brugge, M. (2007). Intraspecific variation in avian pectoral muscle mass: constraints on maintaining manoeuvrability with increasing body mass. Funct. Ecol. 21, 317-326. https://doi.org/10.1111/j.1365-2435.2006.01234.x

Ellington, C. (1991). Limitations on animal flight performance. J. Exp. Biol. 160, 71-91.

Goslow Jr, G., Dial, K. and Jenkins Jr, F. (1990). Bird flight: insights and complications. BioScience 40, 108-115. https://doi.org/10.2307/1311343

Groom, D.J., Toledo, M.C.B., Powers, D.R., Tobalske, B.W. and Welch, K.C. (2018). Integrating morphology and kinematics in the scaling of hummingbird hovering metabolic rate and efficiency. Proc. Royal Soc. B 285, 20172011. https://doi.org/10.1098/rspb.2017.2011.

Hedenström, A. (2002). Aerodynamics, evolution and ecology of avian flight. Trends Ecol. Evol. 17, 415-422. https://doi.org/10.1016/S0169-5347 (02)02568-5

Kamil, B. (2013). MuMIn: multi-model inference. $R$ package version 1.9.0. http://CRAN.Rproject.org/package $=$ MuMIn .

Karasov, W.H. and McWilliams, S.R. (2005). Digestive constraints in mammalian and avian ecology. In J.M. Starck and T. Wang (Eds.), Physiological and ecological adaptations to feeding in vertebrates (pp. 87-112). Enfield, NH: Science Publishers. 
Dial, K.P. (2003). Evolution of avian locomotion: correlates of flight style, locomotor modules, nesting biology, body size, development, and the origin of flapping flight. Auk, 120, 941-952. https://doi.org/10.1642/00048038(2003)120[0941:EOALCO]2.0.CO;2

Kruyt, J.W., Quicazánrubio, E.M., Heijst, G.J.F.V., Altshuler, D.L. and Lentink, D. (2014). Hummingbird wing efficacy depends on aspect ratio and compares with helicopter rotors. J. Royal Soc. Interface 11, 20140585-20140585. https://doi.org/10.1098/rsif.2014.0585

Lee, M.S., Cau, A., Naish, D. and Dyke, G.J. (2014). Sustained miniaturization and anatomical innovation in the dinosaurian ancestors of birds. Science. 345, 562-566. https://doi.org/10.1126/science.1252243

Lind J. (2011). Escape flight in moulting tree sparrows (Passer montanus). Funct. Ecol. 15, 29-35.

Lind J, and Jakobsson S. (2011). Body building and concurrent mass loss: flight adaptations in tree sparrows. Proc. R. Soc. B. 268, 1915-1919. 10.1098/rspb.2001.1740

Louchart, A., and Viriot, L. (2011). From snout to beak: the loss of teeth in birds. Trends Ecol. Evol., 26, 663-673. https://doi.org/10.1016/j.tree.2011.09.004

Marden, J. H. (1987). Maximum lift production during takeoff in flying animals. J. Exp. Biol., 130, 235-258.

Mónus, F., Szabó, K.L., A. and Pénzes, Z. (2011). Intersexual size and plumage differences in tree sparrows (Passer montanus) - a morphological study based on molecular sex determination. Acta Zool. Acad. Sci. Hung. 57, 269-276.

Nespolo, R.F., González-Lagos, C., Solano-Iguaran, J.J., Elfwing, M., Garitano-Zavala, A., Mañosa, S. and Altimiras, J. (2018). Aerobic power and flight capacity in birds: a phylogenetic test of the heart-size hypothesis. J. Exp. Biol. 221, jeb175208. https://doi.org/10.1242/jeb.162693

Piersma, T. and Dietz, M.W. (2007). Twofold seasonal variation in the supposedly constant, species-specific, ratio of upstroke to downstroke flight muscles in red knots Calidris canutus. J. Avian Biol. 38, 536-540. https://doi.org/10.1111/j.2007.0908-8857.04253.x

Pinheiro, J., Bates, D., DebRoy, S., Sarkar, D. and Team, R.C. (2015). nlme: Linear and nonlinear mixed effects 
models. $R$ package version 3.1-122. 2015. http://CRAN.Rproject.org/package=nlme.

353

354

355

356

357

358

359

360

361

362

363

364

365

366

367

368

369

370

371

372

373

374

Plateau, F. (1865). Sur la force musculaire des insects. Bull. Acad. R. Belg. Cl. Sci. 34, 732-757.

Puttick, M.N., Thomas, G.H. and Benton, M.J. (2014). High rates of evolution preceded the origin of birds. Evolution 68, 1497-1510. https://doi.org/10.1111/evo.12363

Rosseel, Y. (2012). lavaan: An R Package for Structural Equation Modeling. J. Stat. Softw., 48, 1-36. http://www.jstatsoft.org/v48/i02/.

Ryeland, J., Weston, M.A. and Symonds, M.R.E. (2017). Bill size mediates behavioural thermoregulation in birds. Funct. Ecol. 31, 885-893. https://doi.org/10.1111/1365-2435.12814

Suarez, R., Staples, J., Lighton, J. and West, T. (1997). Relationships between enzymatic flux capacities and metabolic flux rates: nonequilibrium reactions in muscle glycolysis. Proc. Natl. Acad. Sci. USA 94, 70657069. https://doi.org/10.1073/pnas.94.13.7065

Schielzeth, H. (2010). Simple means to improve the interpretability of regression coefficients. Methods Ecol Evol, 1, 103-113. https://doi.org/10.1111/j.2041-210X.2010.00012.x

Sun, Y., Ren, Z., Wu, Y., Lei, F., Dudley, R. and Li, D. (2016). Flying high: limits to flight performance by sparrows on the Qinghai-Tibet Plateau. J. Exp. Biol. 219, 3642-3648. http://doi.org/ 10.1242/jeb.142216

Sun, Y., Li, M., Song, G., Lei, F., Li, D. and Wu, Y. (2017). The role of climate factors in geographic variation in body mass and wing length in a passerine bird. Avian Res. 8, 1. https://doi.org/10.1186/s40657-016-0059-9

Tattersall, G.J., Arnaout, B. and Symonds, M.R. (2017). The evolution of the avian bill as a thermoregulatory organ. Biol. Rev. Camb. Philos. Soc. 92, 1630-1656. https://doi.org/10.1111/brv.12299

Wright, N.A., Gregory, T.R. and Witt, C.C. (2014). Metabolic 'engines' of flight drive genome size reduction in birds. Proc. R. Soc. B. 281, 20132780. https://doi.org/10.1098/rspb.2013.2780 


\section{Figure legends}

376 Figure 1: The relationships among morphological parameters and their effects on total lifted load for

377 Eurasian tree sparrows (Passer montanus) in the best-fit structural equation model. Total lifted load was

378 positively correlated with wing area, flight muscle mass, and stomach mass, and negatively correlated with bill

379 length. RMSEA, root mean square error of approximation; SRMR, standard root mean square residual; CFI,

380 comparative fit index. *, $\mathrm{p}<0.05 ; * *, \mathrm{p}<0.01$; and $* * *, \mathrm{p}<0.001$. 


\section{Table 1 (on next page)}

Measured variables for morphology, internal anatomy, kinematics, and load-lifting performance for Eurasian tree sparrows (Passer montanus) as averaged for the two sexes, and their correlations with total lifted load (Lifted mass + body mass). 
1 Table 1. Measured variables for morphology, internal anatomy, kinematics, and load-lifting 2 performance for Eurasian tree sparrows (Passer montanus) as averaged for the two sexes, and their 3 correlations with total lifted load (Lifted mass + body mass).

4

\begin{tabular}{|c|c|c|c|c|c|}
\hline \multirow[t]{2}{*}{$\begin{array}{l}\text { Category of } \\
\text { variable }\end{array}$} & \multirow[t]{2}{*}{ Variable } & \multirow[t]{2}{*}{ Mean } & \multirow[t]{2}{*}{ SD } & \multicolumn{2}{|c|}{$\begin{array}{l}\text { Correlation with } \\
\text { total lifted load }\end{array}$} \\
\hline & & & & $r$ & $r^{2}$ \\
\hline \multirow[t]{4}{*}{ Morphology } & Body mass (g) & 19.216 & 1.194 & $0.539 * * *$ & 0.291 \\
\hline & Bill length (mm) & 8.558 & 0.497 & -0.051 & 0.003 \\
\hline & Wing area $\left(\mathrm{cm}^{2}\right)$ & 80.678 & 6.581 & $0.362 * *$ & 0.131 \\
\hline & Aspect ratio & 22.289 & 2.106 & -0.094 & 0.009 \\
\hline \multirow[t]{6}{*}{ Internal anatomy } & Flight muscle mass (g) & 2.450 & 0.250 & $0.669 * * *$ & 0.448 \\
\hline & Heart mass $(\mathrm{g})$ & 0.187 & 0.026 & $0.376 * *$ & 0.141 \\
\hline & Lung mass (g) & 0.097 & 0.017 & $0.258 *$ & 0.067 \\
\hline & Liver mass (g) & 0.345 & 0.061 & $0.264 *$ & 0.07 \\
\hline & Stomach mass (g) & 0.323 & 0.075 & $0.325 * *$ & 0.106 \\
\hline & Gut length (mm) & 136.395 & 7.297 & $0.262 *$ & 0.069 \\
\hline \multirow[t]{2}{*}{ Kinematics } & Wingbeat frequency $(\mathrm{Hz})$ & 5.154 & 0.486 & 0.171 & 0.029 \\
\hline & Wing stroke amplitude $\left(^{\circ}\right)$ & 151.389 & 3.704 & 0.079 & 0.006 \\
\hline \multirow{2}{*}{$\begin{array}{l}\text { Load-lifting } \\
\text { performance }\end{array}$} & Maximum load (g) & 26.027 & 4.592 & & \\
\hline & Total lifted load (g) & 45.243 & 5.124 & & \\
\hline
\end{tabular}




\section{Table 2 (on next page)}

Model-averaged statistical results in the best model set (delta AIC $<6$ ) correlating total lifted load by Eurasian tree sparrows (Passer montanus) with morphological, physiological, and kinematic parameters. 
1 Table 2. Model-averaged statistical results in the best model set (delta AIC $<6$ ) correlating total lifted 2 load by Eurasian tree sparrows (Passer montanus) with morphological, physiological, and kinematic 3 parameters.

\begin{tabular}{|l|c|c|c|c|}
\hline Response variable & Estimate & Adjusted SE & $\mathbf{9 5 \%}$ CI & Relative importance \\
\hline (Intercept) & 0.006 & 0.095 & $-0.180,0.191$ & $\mathbf{1 . 0 0}$ \\
\hline Bill length & -0.259 & 0.090 & $-0.436,-0.083$ & $\mathbf{1 . 0 0}$ \\
\hline Flight muscle mass & 0.579 & 0.130 & $0.324,0.834$ & $\mathbf{1 . 0 0}$ \\
\hline Stomach mass & 0.252 & 0.100 & $-0.057,0.447$ & $\mathbf{0 . 9 6}$ \\
\hline Wing area & 0.262 & 0.133 & $0.001,0.523$ & $\mathbf{0 . 7 0}$ \\
\hline Gut length & 0.148 & 0.086 & $-0.021,0.317$ & 0.48 \\
\hline Heart mass & -0.136 & 0.108 & $-0.349,0.076$ & 0.46 \\
\hline Wingbeat frequency & 0.105 & 0.088 & $-0.067,0.277$ & 0.43 \\
\hline Aspect ratio & 0.146 & 0.140 & $-0.129,0.421$ & 0.42 \\
\hline Body mass & 0.131 & 0.117 & $-0.099,0.361$ & 0.33 \\
\hline Lung mass & -0.083 & 0.102 & $-0.283,0.117$ & 0.28 \\
\hline Wing stroke amplitude & 0.053 & 0.087 & $-0.117,0.223$ & 0.25 \\
\hline Sex & -0.050 & 0.217 & $-0.476,0.376$ & 0.25 \\
\hline Liver mass & 0.026 & 0.102 & $-0.173,0.225$ & \\
\hline
\end{tabular}




\section{Table 3 (on next page)}

Model-averaged statistical results in the best model set (delta AIC < 6) correlating total lifted load of Eurasian tree sparrows (Passer montanus) with all selected variables (see Table 2), and with their level-two interactions. 
1 Table 3. Model-averaged statistical results in the best model set (delta AIC $<6$ ) correlating total lifted

2 load of Eurasian tree sparrows (Passer montanus) with all selected variables (see Table 2), and with their

3 level-two interactions.

4

\begin{tabular}{|l|c|c|c|c|}
\hline Variable & Estimate & Adjusted SE & $\mathbf{9 5 \%}$ CI & Relative importance \\
\hline Intercept) & -0.011 & 0.086 & $-0.18,0.158$ & $\mathbf{1 . 0 0}$ \\
\hline Bill length & -0.262 & 0.088 & $-0.434,-0.089$ & $\mathbf{1 . 0 0}$ \\
\hline Flight muscle mass & 0.611 & 0.098 & $0.419,0.803$ & $\mathbf{1 . 0 0}$ \\
\hline Stomach mass & 0.223 & 0.085 & $0.056,0.389$ & $\mathbf{1 . 0 0}$ \\
\hline Wing area & 0.180 & 0.089 & $0.006,0.354$ & 0.69 \\
\hline Gut length & 0.113 & 0.091 & $-0.066,0.291$ & 0.57 \\
\hline Bill length $\times$ Flight muscle mass & 0.142 & 0.095 & $-0.045,0.329$ & 0.38 \\
\hline Flight muscle mass $\times$ Stomach mass & -0.109 & 0.103 & $-0.311,0.092$ & 0.26 \\
\hline Bill length $\times$ Stomach mass & 0.061 & 0.090 & $-0.115,0.238$ & 0.21 \\
\hline Flight muscle mass $\times$ Wing area & -0.040 & 0.085 & $-0.207,0.127$ & 0.20 \\
\hline Stomach mass $\times$ Wing area & -0.035 & 0.087 & $-0.206,0.137$ & 0.20 \\
\hline Bill length $\times$ Wing area & -0.015 & 0.127 & $-0.263,0.234$ & 0.17 \\
\hline Flight muscle mass $\times$ Gut length & 0.065 & 0.097 & $-0.126,0.255$ & 0.15 \\
\hline Bill length $\times$ Gut length & 0.057 & 0.090 & $-0.12,0.233$ & 0.13 \\
\hline Gut length $\times$ Stomach mass & -0.034 & 0.102 & $-0.233,0.166$ & 0.10 \\
\hline Gut length $\times$ Wing area & 0.011 & 0.103 & $-0.192,0.214$ & \\
\hline Variables with relative importance score $>0.7$ are shown in bold type, and were included in further analysis.
\end{tabular}

5 
Figure 1

The relationships among morphological parameters and their effects on total lifted load for Eurasian tree sparrows (Passer montanus) in the best-fit structural equation model.

Total lifted load was positively correlated with wing area, flight muscle mass, and stomach mass, and negatively correlated with bill length. RMSEA, root mean square error of approximation; SRMR, standard root mean square residual; $\mathrm{CFI}$, comparative fit index. ${ }^{*}, \mathrm{p}<$ $0.05 ; * *, p<0.01 ;$ and $* * *, p<0.001$.

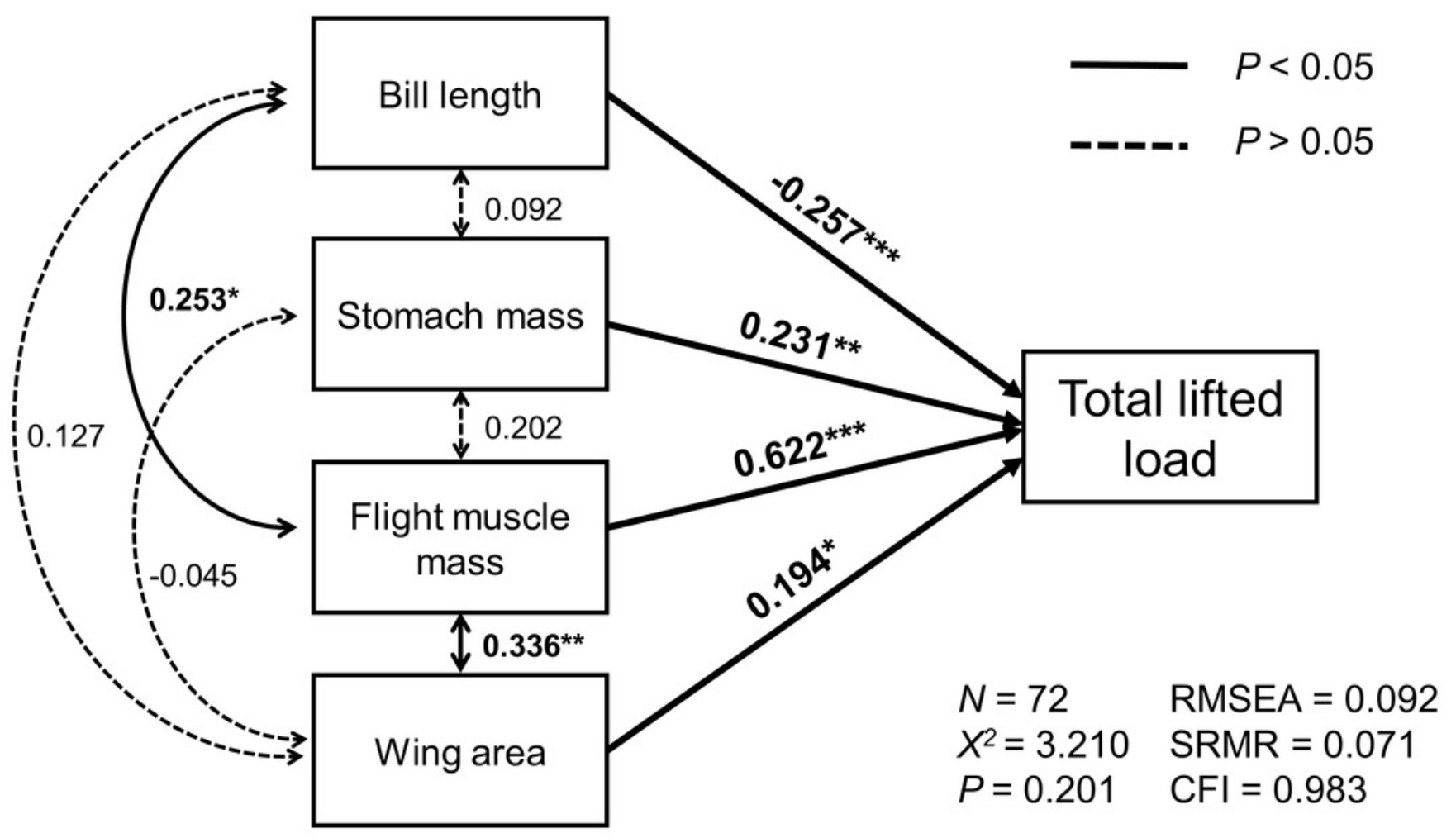

